\title{
Predictors of Heart Attack among Adults Aged 18 Years and Older in the United States
}

\author{
Miranda Perry ${ }^{1}$, MPH, Mechelle D. Claridy, MPH ${ }^{1}$, Stephanie Miles-Richardson, DVM, PhD ${ }^{1}$, \\ Gemechu B. Gerbi, MSc, PhD $^{1^{*}}$ \\ ${ }^{I}$ Morehouse School of Medicine,720 Westview Dr. SW,NCPC Suite 346, Atlanta, GA 30310-1495 USA \\ *Corresponding Author: Gemechu B. Gerbi, MSc, PhD, Morehouse School of Medicine, 720 Westview \\ Dr. SW,NCPC Suite 346, Atlanta, GA 30310-1495 USA, Email: ggerbi@msm.edu
}

\begin{abstract}
Background: According to the American Heart Association, the estimated annual incidence of heart attacks in the United States (US) is 720,000 new attacks and 335,000 recurrent attacks. The objective of this study was to assess predictors of heart attacks among adults aged $\geq 18$ years in the US.

Methods: Data were analyzed from the 2018 Behavioral Risk Factor Surveillance System. Multivariable analyses were used to estimate adjusted odds ratios (AORs) and 95\% confidence intervals (95\% CIs) for factors associated with the prevalence of a heart attack in the US. Analyses were conducted using SAS version 9.4 .

Results: Of the 435,118 respondents, 26,412 (6\%) reported having had a heart attack, while 408,706 (94\%) did not report having had a heart attack. Factors associated with higher odds of reporting having had a heart attack included: being male (AOR 2.50; 95\% CI; 2.38-2.53) and being 25 years or older. Other factors associated with higher odds included having less than a college education and having an annual household income $<\$ 40,000$. Respondents from the Midwest (AOR=1.14; 95\% CI=1.09-1.19), Northeast (AOR=1.10; 95\% CI=1.05-1.16), South (AOR=1.30; 95\% CI=1.24-1.36), and those who did not participate in any physical activities or exercises (AOR=1.47; 95\% CI=1.43-1.52) were also more likely to report having had a heart attack.
\end{abstract}

Conclusion: Significant differences in the risk associated with a heart attack were linked to sociodemographic factors, exercise, and region of residence. Developing targeted interventions, especially for high-risk populations, is needed to reduce the risk for subsequent heart attacks among adults in the US.

Keywords: sociodemographic, exercise, heart attack, adults aged $\geq 18$ years, United States

\section{INTRODUCTION}

Heart disease refers to several types of heart conditions. Many of the problems associated with heart disease are related to the process of atherosclerosis [1]. Atherosclerosis is a condition that develops when plaque builds up in the walls of the arteries [2]. This buildup narrows the arteries, making it harder for blood to flow through [3]. If a blood clot forms, it can block the blood flow, which can cause a heart attack $[4,5]$.

In the United States (US), about 1 million Americans have a heart attack each year [2] and a heart attack happens every 40 seconds $[6,7]$. A heart attack, also called a myocardial infarction, occurs when a part of the heart muscle does not get enough blood and oxygen
[8]. One cause of a heart attack is the narrowing of coronary arteries from a buildup of fat, cholesterol, and other substances that comprise plaque [4]. Heart attacks are a leading cause of death for both genders [2]. Additionally, heart attacks have a major economic cost. In 2013, heart attacks cost 12.1 billion, making this one of the most expensive conditions treated in the US [9]. Heart attacks can have harmful and long-lasting effects, including disability and economic costs not only for people living with the disease but also for their families, workplaces, and communities.

When examining sociodemographic factors and their relationship to myocardial infarction, several common themes emerge. There is often a relationship between where people live, and their incomes, as well as education, race, and 
age $[10,11]$. Persons considered low-income are at higher risk of myocardial infarction, reinfarction, and other health issues [12, 13]. Additionally, there are well-documented disparities in treatment and survival by race, socioeconomic status (SES), age, and gender [14-17]. However, the sociodemographic predictors of heart attack are less studied. Limited studies utilized a nationally representative sample.

An examination of the association of sociodemographic variables is an important addition to the current body of literature regarding myocardial infarction. Such information will address gaps in the literature which mainly focuses on differences in treatment, mortality, and knowledge of myocardial infarction across sociodemographic groups. Importantly, an understanding of the predictors of myocardial infarction will allow for the development of targeted interventions.

\section{METHODS}

\subsection{Data and Study Sample}

Data were analyzed from the 2018 Behavioral Risk Factor Surveillance System (BRFSS), a project between all of the 50 states in the US, the District of Columbia, three US territories, and the Centers for Disease Control and Prevention (CDC) [18]. The BRFSS collects uniform, state-specific data on preventive health practices and risk behaviors that are linked to chronic diseases, injuries, and preventable infectious diseases that affect noninstitutionalized adult population - aged 18 years or older - who reside in the US. The BRFSS is the nation's leading system of healthrelated risk behaviors, chronic health conditions, and usage of preventive services annually [18].

The BRFSS questionnaire is administered on a continuous basis by telephone using randomdigit-dial sampling methods. The design consists of a probability sample of all households with telephones in the state [18]. The BRFSS standardized questionnaires are developed by the CDC and state public health departments to include a standard core, optional modules, and state-added questions [18]. Since the BRFSS data files are publicly available via the website: https://www.cdc.gov/brfss/_data_ documentation/index.htm, data use agreements were not required for this study. Furthermore, as the BRFSS data do not contain personally identifiable information, this study is exempt from the Institutional Review Board review.

\subsection{Measures}

All measures of the study were based on selfreported data obtained from the 2018 BRFSS.

\subsection{Dependent Variable}

The dependent variable was a heart attack. Heart attack was defined using the following question from the 2018 Behavioral Risk Factors Surveillance System: "(Ever told) you had a heart attack, also called a myocardial infarction?" Possible responses included "yes", "no", "don't know/ not sure", and "refused". Only records with "yes" or "no" responses for "ever been told you have had a heart attack' were included in the analysis. Records with "unknown or "refused" responses or missing data were excluded from the analysis to minimize errors in estimation'.

\subsection{Main Independent Variable}

The main independent variable was physical activity. Physical activity was defined using the following question from the 2018 BRFSS: "During the past month, other than your regular job, did you participate in any physical activities or exercises such as running, calisthenics, golf, gardening, or walking for exercise?" Possible responses included "yes," "no", "don't know/not sure", and "refused". However, we only included "yes" or "no" responses in the analysis. Records with "unknown" or "refused" responses or missing data were excluded from the analysis to minimize estimation errors.

\subsection{Covariates}

Control variables included were sociodemographic characteristics, including gender, race or ethnicity, age, education, income, and region of residence. Gender was dichotomized as female and male. Race or ethnicity was categorized as Non-Hispanic White, Non-Hispanic Black, American Indian or Alaskan Native, Asian, Hispanic/Latino, Native American or Pacific Islander, and mixed. Age was categorized as 18-24, 25-34, 35-44, 45-54, $55-64$, and $\geq 65$ years. Income was categorized as $\quad \leq \$ 14,999, \quad \$ 15,000-\$ 24,999, \quad \$ 25,000-$ $\$ 34,999, \$ 35,000-\$ 49,999$, and $\geq \$ 50,000$.

\subsection{Statistical Analysis}

Univariate analysis was performed to provide a description of the frequencies of the study population. Initially, we performed a bivariate analysis to assess factors independently associated with self-reported history of heart attack. We included all variables that had 
achieved $\mathrm{P} \leq .05$ in the bivariate analysis in our final multivariable logistic regression model and obtained the adjusted odds ratios (AORs) and 95\% confidence intervals (95\% CIs). A 2- sided $\mathrm{P}$ value of $\leq .05$ was considered to indicate statistical significance. Analyses were conducted using SAS 9.4 [19].

\section{Results}

Table 1 shows a summary of the demographic, SES, and physical activity characteristics of the participants by number and percentages in relation to their gender, race or ethnicity, age group, level of education, level of income, and

Table1: Number* and percentage of adults who reported having had a heart attack by select characteristics: 2018 BRFSS, United States perceived physical activity participation. Of the 435,118 participants who responded to the question: "(Ever told) you had a heart attack, also called a myocardial infarction?", 26412 $(6 \%)$ reported a history of heart attack, and 408706 (94\%) reported no history of heart attack (Table 1). The majority of respondents who reported a history of heart attack were males $(8.0 \%), 65$ or older $(11.1 \%)$, multi-race $(7.4 \%)$, had an income less than $\$ 15,000$ $(10.8 \%)$, had an elementary education $(11.7 \%)$, resided in the south $(7.0 \%)$, and did not participate in physical activity $(9.5 \%)$.

\begin{tabular}{|c|c|c|c|c|}
\hline \multirow[t]{2}{*}{ Select characteristics } & \multicolumn{4}{|c|}{$\begin{array}{c}\text { Self-Reported Heart Attack } \\
(\mathrm{N}=\mathbf{4 3 5 , 1 1 8 )}\end{array}$} \\
\hline & Yes & No & Total & p-value \\
\hline & $\mathrm{n}(\%)$ & n (\%) & n (\%) & \\
\hline & $26412(6)$ & $408706(94)$ & $435118(100)$ & \\
\hline Gender & & & & $<.0001$ \\
\hline Female & $10631(5)$ & $227284(95)$ & $237915(100)$ & \\
\hline Male & $15692(8)$ & $180479(92)$ & $196171(100)$ & \\
\hline Age Group & & & & $<.0001$ \\
\hline 18 to 24 & $118(1)$ & $25779(99)$ & $25897(100)$ & \\
\hline 25 to 34 & $368(1)$ & $46067(99)$ & $46435(100)$ & \\
\hline 35 to 44 & $901(2)$ & $51366(98)$ & $52267(100)$ & \\
\hline 45 to 54 & $2385(4)$ & $65079(96)$ & $67464(100)$ & \\
\hline 55 to 64 & $5732(6)$ & $84419(94)$ & $90151(100)$ & \\
\hline 65 or older & $16908(11)$ & $135996(89)$ & $152904(100)$ & \\
\hline Race and ethnicity & & & & $<.0001$ \\
\hline Multiracial & $626(7)$ & $7838(93)$ & $8464(100)$ & \\
\hline White, Non-Hispanic & $20550(6)$ & $302941(94)$ & $323491(100)$ & \\
\hline Black, Non-Hispanic & $1916(5)$ & $33745(95)$ & $35661(100)$ & \\
\hline Hispanic & $1431(4)$ & $35272(96)$ & $36703(100)$ & \\
\hline Other & $1335(6)$ & $21194(94)$ & $22529(100)$ & \\
\hline Annual Household Income & & & & $<.0001$ \\
\hline Less than $\$ 15,000$ & $3724(11)$ & $30661(89)$ & $34385(100)$ & \\
\hline$\$ 15,000-\$ 24,999$ & $5277(9)$ & $52381(91)$ & $57658(100)$ & \\
\hline$\$ 25,000-\$ 34,999$ & $2746(7)$ & $34886(93)$ & $37632(100)$ & \\
\hline$\$ 35,000-\$ 49,999$ & $3120(6)$ & $46253(94)$ & $49373(100)$ & \\
\hline More than $\$ 50,000$ & $6726(4)$ & $174037(96)$ & $180763(100)$ & \\
\hline Level of Education & & & & $<.0001$ \\
\hline Elementary level & $1205(12)$ & $9057(88)$ & $10262(100)$ & \\
\hline Less than High School & $49(7)$ & $607(93)$ & $656(100)$ & \\
\hline High School Graduate & $8681(7)$ & $109538(93)$ & $118219(100)$ & \\
\hline Some College/ Technical School & $9645(7)$ & $131054(93)$ & $140699(100)$ & \\
\hline College Graduate & $6749(4)$ & $157023(96)$ & $163772(100)$ & \\
\hline Region of residence & & & & $<.0001$ \\
\hline Midwest & $6818(6)$ & $104195(94)$ & $111013(100)$ & \\
\hline North & $5304(6)$ & $85471(94)$ & $90775(100)$ & \\
\hline South & $8262(7)$ & $110229(93)$ & $118491(100)$ & \\
\hline West & $4657(5)$ & $87361(95)$ & $92018(100)$ & \\
\hline Exercise Status & & & & $<.0001$ \\
\hline Yes & $15947(5)$ & $309195(95)$ & $325142(100)$ & \\
\hline No & $10396(10)$ & $98956(90)$ & $109352(100)$ & \\
\hline
\end{tabular}

Note: *Frequencies may vary due to missing values 
Table 2 captures the results of multivariable analyses showing the likelihood of participants having history of a heart attack. Factors associated with higher odds of reporting a heart attack included: being male (AOR 2.50; 95\% CI; 2.38-2.53) and being in the age groups 25 and above. Other factors associated with higher odds included having less than a college education and having an annual household

Table2: Multivariable results for adults having had a heart attack by select characteristics: 2018 BRFSS, United States

\begin{tabular}{|c|c|c|}
\hline \multirow[t]{2}{*}{ Select Characteristics } & \multicolumn{2}{|c|}{ Ever had a heart attack $(N=26,412)$} \\
\hline & AOR & $95 \% \mathrm{CI}$ \\
\hline \multicolumn{3}{|l|}{ Gender } \\
\hline Female & REF & \\
\hline Male & 2.45 & $2.38-2.53$ \\
\hline \multicolumn{3}{|l|}{ Age Group } \\
\hline $18-24$ & REF & \\
\hline $25-34$ & 2.04 & $1.60-2.65$ \\
\hline $35-44$ & 4.88 & $3.89-6.22$ \\
\hline $45-54$ & 9.82 & $7.89-12.46$ \\
\hline $55-64$ & 17.81 & $14.35-22.53$ \\
\hline 65 or older & 33.14 & $26.74-41.87$ \\
\hline \multicolumn{3}{|l|}{ Race and ethnicity } \\
\hline White, Non-Hispanic & REF & \\
\hline Multi-racial & 1.45 & $1.31-1.59$ \\
\hline Black, Non-Hispanic & 0.74 & $0.70-0.78$ \\
\hline Hispanic & 0.80 & $0.74-0.86$ \\
\hline Other & 1.18 & $1.10-1.26$ \\
\hline \multicolumn{3}{|l|}{ Annual Household Income } \\
\hline Less than $\$ 15,000$ & 2.87 & $2.73-3.01$ \\
\hline$\$ 15,000-\$ 24,999$ & 2.20 & $2.11-2.30$ \\
\hline$\$ 25,000-\$ 34,999$ & 1.67 & $1.58-1.75$ \\
\hline$\$ 35,000-\$ 49,999$ & 1.48 & $1.41-1.55$ \\
\hline More than $\$ 50,000$ & REF & \\
\hline \multicolumn{3}{|l|}{ Level of Education } \\
\hline Elementary level & 1.68 & $1.54-1.84$ \\
\hline Less than High School & 1.16 & $0.75-1.71$ \\
\hline High School Graduate & 1.31 & $1.26-1.37$ \\
\hline Some college & 1.39 & $1.34-1.45$ \\
\hline College Graduate & REF & \\
\hline \multicolumn{3}{|l|}{ Region of Residence } \\
\hline West & REF & \\
\hline Midwest & 1.14 & $1.09-1.19$ \\
\hline North & 1.10 & $1.05-1.16$ \\
\hline South & 1.30 & $1.24-1.36$ \\
\hline \multicolumn{3}{|l|}{ Exercise Status } \\
\hline Yes & REF & \\
\hline No & 1.47 & $1.43-1.52$ \\
\hline
\end{tabular}

Note: $\mathrm{AOR}=$ adjusted odds ratio; $\mathrm{CI}=$ confidence interval

\section{DISCUSSION}

Overall, $6 \%$ of the respondents in this analysis reported having a history of heart attack. The prevalence of myocardial infarction among adults at the national level are less studied. However, a previous study found that, between 2001 and 2012, the prevalence of myocardial income $<\$ 40,000$. Respondents from the Midwest $\quad(\mathrm{AOR}=1.14 ; \quad 95 \% \quad \mathrm{CI}=1.09-1.19)$, Northeast $\quad(\mathrm{AOR}=1.10 ; 95 \% \quad \mathrm{CI}=1.05-1.16)$, South $(\mathrm{AOR}=1.30 ; 95 \% \mathrm{CI}=1.24-1.36)$, and those who did not participate in any physical activities or exercises (AOR $=1.47 ; 95 \%$ $\mathrm{CI}=1.43-1.52$ ) were also more likely to report a heart attack. 
result, many may not have sought care from a physician nor received a diagnosis of myocardial infarction. Furthermore, about 1 in 5 heart attacks are silent which means the person is unaware [22].

In the current study, men were more likely to report a history of heart attack. Prior research demonstrated that men were more likely to have at least one of three known cardiovascular risk factors - uncontrolled high blood pressure, uncontrolled high levels of low-density lipoproteins cholesterol, and current smoking [7]. Additionally, differences in the presentation, pathophysiological, and outcome in patients with acute myocardial infarction were present according to gender [16]. Heart attacks and Coronary Heart Disease are less studied in women. Since 1984, the recorded mortality rate has been greater for men than women [16]. Furthermore, women were less likely to be treated with guideline-based recommendations leading to worse outcomes, including recurrent heart attacks and death [16]. A possible reason for women having a lower reported prevalence of heart attack is that women are more likely to have uncommon symptoms and may not know that they are experiencing a heart attack [8].

The current study found that respondents who were older were more likely to have history of a heart attack. This is consistent with previous research on the topic. People aged 65 and older are more likely than younger people to be diagnosed with a heart attack [20, 23]. Aging can cause changes in the heart and blood vessels and increase a person's risk of heart disease [2, 3]. A major cause of heart attack and heart disease is the buildup of fatty deposits in the walls of arteries over many years. A buildup of plaque can harden and narrow arteries which limits the flow of oxygen-rich blood to the organs and body [4]. Plaque buildup in the coronary arteries of the heart muscle can reduce the flow of blood to the heart, resulting in heart attack [3]. Heart attacks limit the activity and erode the quality of life of millions of older adults [3].

The current study found that Non-Hispanic Black respondents were less likely to report a history of a heart attack. These findings are not consistent with previous literature. NonHispanic Blacks have been found to have a greater burden of stroke, myocardial infarction, and other cardiovascular events $[14,15]$. NonHispanic Black patients experience worse outcomes than Non-Hispanic White patients following acute myocardial infarction [14, 15]. Non-Hispanic Black and Hispanic myocardial infarction patients are more likely to present with comorbidities, experience longer delays before treatment, and suffer worse outcomes than non- Hispanic White patients [14, 15]. Non-Hispanic Black and Hispanic myocardial patients are more likely to be younger, female, or have hypertension or diabetes than nonHispanic White patients [14]. One potential reason for the discrepancy between findings in the current study and previous research is that we used self-reported data of a history of heart attack. As a result, the current study does not account for people who die from a heart attack. Because Non- Hispanic Black patients are more likely to die from heart attack, the current study may include an underestimation in heart attack rates by race. Without consistent documentation of heart attack disparities, it may be difficult to identify and tailor interventions for demographic groups most affected by the disease.

The current study found that being of a lower income was associated with a history of heart attack. Similarly, prior research has demonstrated an association between income inequality in the United States and heart attacks [24]. In addition to overall income inequality having an impact on heart attack rates, childhood SES contributes to heart attack prevalence in the United States. However, childhood SES influences the risk of heart attacks for women but not men [25]. Previously, data from the Jackson Heart Study demonstrated that there was an inverse association between SES and the burden of cardiovascular disease in Non-Hispanic Blacks, which is a similar association found in the general population [17]. The burden of cardiovascular disease in NonHispanic Blacks may be reduced by improved SES [17]. People who are considered lowincome are at a higher risk for heart attack due to social determinants such as lack of access to healthcare and preventive services, limited access to healthy foods, inability to exercise safely, and economic and work-related stress $[10,26]$.

Similar to previous studies, we found that individuals with less than a college education reported higher levels of heart attack [10, 12]. Previous research has also suggests that persons with lower levels of education had higher 1-5year mortality rates after a myocardial infarction [12]. Lower education may be related to a higher prevalence of myocardial infarction for 
several reasons. First, people who have lower levels of education tend to have a lower income [12]. Lower income may decrease one's access to healthcare services, healthful foods, and safe places to exercise. Additionally, people of a lower education level may be less knowledgeable about health risks.

The current study found higher rates of reported heart attack in the South. A recent study found that hotspots for cardiovascular disease were in primarily in the southeastern region of the US [27]. Data from the CDC Interactive Atlas show a concentration of heart attacks in the southern region of the US [11]. Similarly, other researchers have found a higher prevalence of cardiovascular diseases and stroke in this region of the US [28]. Potential explanations for these consistent findings are related to dietary differences, limited access to healthcare, and regional differences in public health policies [27].

The current study found that exercise lowers one's risk of a heart attack. Lifestyle choices which include no smoking, not being obese, having regular physical activity, and consuming a healthy diet are associated with a decreased risk of coronary artery disease [29]. Because lifestyle factors reduce the risk of coronary artery disease and coronary artery disease is a major cause of heart attacks, it can be inferred that the lifestyle modifications including adding physical activity will reduce the risk of heart attacks. Lifestyle modifications such as smoking cessation, lowering the cholesterol level, medical management of diagnosed hypertension, maintenance of a physically active lifestyle, avoidance of obesity, maintenance of normal glucose levels, and taking low-dose aspirin $[30,31]$ have all been shown to reduce the likelihood of coronary artery disease and heart attacks.

\subsection{Study Limitations}

This study has certain limitations. First, the BRFSS excludes individuals without telephone services, those on military bases, and individuals in institutions. For this reason, generalizability to the entire US population is limited. Second, since the BRFSS is a phone survey answered by each individual participant, the answers to some of the questions may be subject to recall or reporting bias. Third, with respect to heart attack, the BRFSS asks "(Ever told) you had a heart attack, also called myocardial infarction?". This limits our ability to make strong statements about the direction of the association between history of heart attack, exercise, and demographic characteristics. Utilizing this question can cause us to miss people who do not know they had a heart attack as well as people who died from a heart attack.

\section{CONCLUSiON}

We conclude that exercise and demographic variables (gender, income, and education) influence the self-reported history of heart attack in adults in the US. These findings indicate the need for preventive and treatment strategies for people who are more at risk for heart attack. The reported variability between some of our findings and previous studies suggests that more research is needed to provide more accurate estimates of the prevalence of heart attack in specific demographics.

\section{Clinical IMPLiCATIONS RECOMMENDATIONS}

The findings of this study can be used to target specific populations in terms of gender, age, race/ethnicity, income level, education level, region of residence and exercise to implement programs tailored to their specific needs and goals. For instance, being male, being in the age groups 25 and above, low SES, respondents from the Midwest, Northeast, South, and those who did not participate in any physical activities or exercises had an increased likelihood of reporting a heart attack. Thus, to reduce heart attacks, programs or interventions can be developed to target males with these characteristics. Utilizing data such as that reported in this study, can assist in addressing these public health problems and may ultimately reduce the need for health care spending, increase life expectancy due to better health, and prevent or alleviate other illnesses and chronic diseases in the US population. Future studies to further understand the relationship of SES, region of residence and exercise are critical, and may lead to our ability to reduce the prevalence of heart attacks in adults in the US. Future studies should also include qualitative methods to determine community specific characteristics that increase the risk of heart attack.

\section{REFERENCES}

[1] American Heart Association. What is a Heart Attack. n.d. [cited 2020 February 15]; Available from: https://www.heart.org/en/ health-topics/heart-attack/about-heart-attacks.

[2] National Institute on Aging. What is a Heart Attack? n.d. June 2018 [cited 2020 February 
10]; Available from: https://www.nia.nih.gov/ health/what-heart-attack.

[3] National Institute on Aging. Heart Health and Aging. n.d. [cited 2020 February 17]; Available from: https://www.nia.nih.gov/ health/heart-health-and-aging.

[4] American Heart Association. About Heart Attacks. n.d. [cited 2020 February 20]; Available from: https://www.heart.org/en/ health-topics/heart-attack/about-heart-attacks.

[5] Centers for Disease Control and Prevention. About Heart Disease. n.d. [cited 2020 February 19]; Available from: https://www.cdc.gov/ heartdisease/about.htm.

[6] Benjamin, E.J., et al., Heart disease and stroke statistics-2019 update: a report from the American Heart Association. Circulation, 2019. 139(10): p. e56-e528.

[7] Fryar, C.D., Te-Ching Chen, and Xianfen Li, Prevalence of uncontrolled risk factors for cardiovascular disease: United States, 19992010. National Center for Health Statisitics, 2012.

[8] Centers for Disease Control and Prevention. Heart Attack Symptoms, Risk, and Recovery. n.d. [cited 2020 February 17]; Available from: https://www.cdc.gov/heartdisease/heart_attack. htm.

[9] American Heart Association. Heart Disease and Stroke Statisitics 2018 At-a-Glance. 2018 [cited 2020 February 15]; Available from: https://healthmetrics.heart.org/wp-content/ uploads/2018/02/At-A-Glance-Heart-Diseaseand-Stroke-Statistics-2018.pdf.

[10] Coughlin, S.S. and L. Young, Social Determinants of Myocardial Infarction Risk and Survival: A Systematic Review. European journal of cardiovascular research, 2020. 1(1).

[11] Centers for Disease Control and Prevention. Interactive Atlas of Heart Disease and Stroke. n.d. [cited 2020 November 1]; Available from: https://www.cdc.gov/dhdsp/maps/atlas/index.ht m.

[12] Coady, S.A., et al., Individual education, area income, and mortality and recurrence of myocardial infarction in a Medicare cohort: the National Longitudinal Mortality Study. BMC Public Health, 2014. 14(1): p. 1-11.

[13] Kucharska-Newton, A.M., et al., Socioeconomic indicators and the risk of acute coronary heart disease events: comparison of population-based data from the United States and Finland. Annals of epidemiology, 2011. 21(8): p. 572-579.

[14] Graham, G., Disparities in cardiovascular disease risk in the United States. Current cardiology reviews, 2015. 11(3): p. 238-245.

[15] Graham, G.N., et al., Racial disparities in patient characteristics and survival after acute myocardial infarction. JAMA network open, 2018. 1(7): p. e184240-e184240.

[16] Mehta, L.S., et al., Acute myocardial infarction in women: a scientific statement from the American Heart Association. Circulation, 2016. 133(9): p. 916-947.

[17] Min, Y.I., et al., Cardiovascular disease burden and socioeconomic correlates: findings from the Jackson Heart Study. Journal of the American Heart Association, 2017. 6(8): p. e004416.

[18] Centers for Disease Control and Prevention. Behavioral Risk Factors Surveillance System. n.d. [cited 2020 February 8]; Available from: https://www.cdc.gov/brfss/index.html.

[19] SAS Institute. Cary, NC, USA.

[20] Yoon, S.S.S., et al., Trends in the prevalence of coronary heart disease in the US: National Health and Nutrition Examination Survey, 2001-2012. American journal of preventive medicine, 2016. 51(4): p. 437-445.

[21] Patel, A., et al., Awareness of heart attack signs and symptoms and calling 9-1-1 among US adults. Journal of the American College of Cardiology, 2018. 71(7): p. 808-809.

[22] Centers for Disease Control and Prevention. Heart Disease Facts. 2019 [cited 2020 Februray 23]; Available from: https://www.cdc.gov/ heartdisease/facts.htm.

[23] Huma, S., et al., Modifiable and non-modifiable predisposing risk factors of myocardial infarction-A review. Journal of pharmaceutical sciences and research, 2012. 4(1): p. 1649.

[24] Pabayo, R., I. Kawachi, and S.E. Gilman, US State-level income inequality and risks of heart attack and coronary risk behaviors: longitudinal findings. Int J Public Health, 2015. 60(5): p. 573-88.

[25] Hamil-Luker, J. and A.M. O'Rand, Gender differences in the link between childhood socioeconomic conditions and heart attack risk in adulthood. Demography, 2007. 44(1): p. 137-58.

[26] Havranek, E.P., et al., Social Determinants of Risk and Outcomes for Cardiovascular Disease: A Scientific Statement From the American Heart Association. Circulation, 2015. 132(9): p. 873-98.

[27] Sun, W., F. Gong, and J. Xu, Individual and contextual correlates of cardiovascular diseases among adults in the United States: a geospatial and multilevel analysis. GeoJournal, 2020. 85(6): p. 1685-1700.

[28] Esenwa, C., et al., Historical slavery and modern-day stroke mortality in the United States stroke belt. Stroke, 2018. 49(2): p. 465469.

[29] Dimovski, K., M. Orho-Melander, and I. Drake, A favorable lifestyle lowers the risk of coronary 
artery disease consistently across strata of nonmodifiable risk factors in a population-based cohort. BMC Public Health, 2019. 19(1): p. 1575.

[30] Åkesson, A., et al., Low-risk diet and lifestyle habits in the primary prevention of myocardial infarction in men: a population-based prospective cohort study. Journal of the American college of cardiology, 2014. 64(13): p. 1299-1306.

[31] Manson, J.E., et al., The primary prevention of myocardial infarction. New England journal of medicine, 1992. 326(21): p. 1406-1416.

Citation: Gemechu B. Gerbi, MSc, PhD, et.al. Predictors of Heart Attack among Adults Aged 18 Years and Older in the United States. ARC Journal of Public Health and Community Medicine. 2021; 6(2):1-8. Doi:doi.org/10.20431/2456-0596.0602001.

Copyright: (C) 2021 Authors. This is an open-access article distributed under the terms of the Creative Commons Attribution License, which permits unrestricted use, distribution, and reproduction in any medium, provided the original author and source are credited. 